\title{
Solving a Geometrical Locus Problem Using GeoGebra Software and Then Analytical Geometry
}

\author{
M. El Aydi'1,2*, R. Bendaoud1, M. Sbaa ${ }^{3}$, M. Barkatou', M. Benhmida1 \\ ${ }^{1}$ Department of Physics, Faculty of Sciences, U.C.D, EL Jadida, Morocco \\ ${ }^{2}$ Mathematics Department, Regional Centre of the Jobs of Education and Training, El Jadida, Morocco \\ ${ }^{3}$ Departement of Mathematics, Faculty of Sciences, Settat, Morocco \\ ${ }^{4}$ Department of Mathematics, Faculty of Sciences, U.C.D, EL Jadida, Morocco \\ Email: ^elaydi58@gmail.com
}

How to cite this paper: El Aydi, M. Bendaoud, R., Sbaa, M., Barkatou, M. and Benhmida, M. (2020) Solving a Geometrical Locus Problem Using GeoGebra Software and Then Analytical Geometry. Open Access Library Journal, 7: e6539.

https://doi.org/10.4236/oalib.1106539

Received: June 19, 2020

Accepted: July 14, 2020

Published: July 17, 2020

Copyright $\odot 2020$ by author(s) and Open Access Library Inc.

This work is licensed under the Creative Commons Attribution International License (CC BY 4.0).

http://creativecommons.org/licenses/by/4.0/

\begin{abstract}
In this work, intended for future teachers, we present a solution of a geometric locus problem using the GeoGebra software. This allows first to visualize the shape of the geometric locus, then to state a conjecture to validate a solution founded by analytical geometry methods. The search for a geometric locus using GeoGebra software makes the activity more attractive.
\end{abstract}

\section{Subject Areas \\ Didactics of Mathematics}

\section{Keywords}

Open Source Software, GeoGebra, Orthocenter, Analytical Geometry

\section{Introduction}

Several countries have made significant investments in information and communication technology to ensure effective classroom teaching and learning. According to Fluck (2010), information and communication technologies should play a transformative role in education rather than integration into existing disciplines. One might ask what new teaching methods are suitable for students using such new tools [1] [2].

The purpose of studying geometrical locus problems is to determine a set of points satisfying given geometric construction conditions.

This allows students to apply geometry; algebra and analysis tools learned 
during their straining. Problems of geometric locus can be seen from the college by using for example the property of the center of gravity of a triangle. In classes of the secondary qualifying, the pupils essentially use the bary center, the scalar product, or the transformations of the plane, at the Faculty of Science, students use functions, parametric coordinates, polar coordinates, etc.

Unfortunately, teachers generally do not address geometric locus issues with theirs students.

Our trainees (future teachers) rarely make use of analytical geometry, but they experience joy using dynamic geometry software like GeoGebra.

\section{Brief Description of the GeoGebra software}

It is a dynamic geometry software, with which one can make figures then explore, verify and study their properties by discovering many cases.

\subsection{Advantages of using GeoGebra}

Using GeoGebra has several advantages:

- Ease of use: GeoGebra is a simple and easy to use interface.

- Multifunctionality: GeoGebra is a multi-functional software package.

- Free software: that is to say, that the user can download and use for free and "open source" that the user has the right to make modifications.

- You can find many helps on the software site:

- http://www.geogebra.org/cms/index.php?option=com content\&task=blogcat egory\&id $=75 \&$ Itemid $=61$

- A handling document: http://www.geogebra.org/help/geogebraquikstat fr.pdf

\subsection{Implementation: Basic Functionalities and Main Areas of GeoGebra Software}

- The input bar: it allows to enter commands and thus directly define objects; for example: $f(x)=x^{4}-2 x^{2}$ Figure 1 .

- The toolbar: there are the main construction tools (create point, line, perpendicular line, polygon, circle, angle, etc.); for example: point on object $D$, extrema $A, B$ and $C$, the point $E$ in the middle of $[B D]$, in red, the geometric locus of $E$ when the point $D$ varies Figure 1 .

- The algebra window: there is information on the objects created (coordinates, equations, length, etc.); for example: the function $f$.

- The worksheet: The worksheet: objects are created and represented graphically, for example the function $f$ and its graph.

- The spreadsheet allows calculations, statistics and the construction of diagrams and graphs.

The work area of the GeoGebra window shows the geometric aspect of an object and the algebra area shows its analytical aspect, which makes it easier for students to acquire a double perception of a problem. 


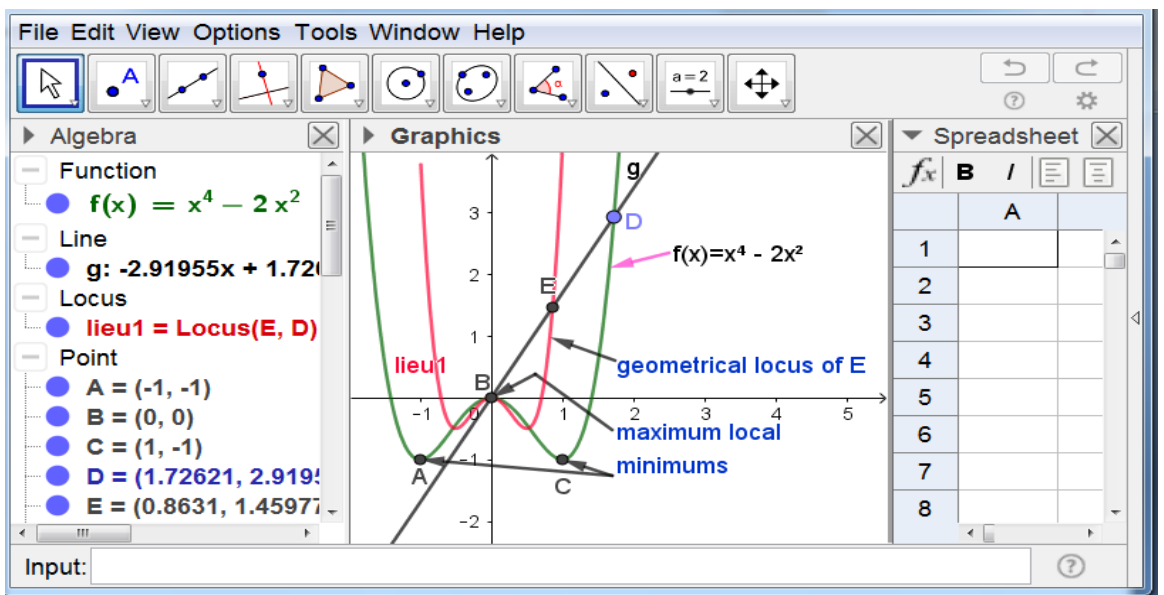

Figure 1. Main areas of GeoGebra software.

Thus, the cursor tool allows to create variables or parameters, to deal with a mathematical problem or a problem from everyday life [3].

\section{Research Objectives}

Consider the following problem: "In the plane, $A B C$ is a triangle of orthocenter $H$. Determine the geometrical locus $\mathrm{L}$ of the points $H$ when $C$ moves on a fixed line $(D)$ ".

This problem of Geometry was proposed for two hours to 100 future teachers graduated in mathematics or physics., With the exception of two students, they could not solve the problem and they did not think to use analytical geometry. When the problem was solved collectively, I started by using the Geogebra software, the teacher trainees showed a real enthusiasm. The use of GeoGebra software helped to deal with the problem using analytical geometry. This finding is consistent with the studies carried out by Hennessy, Fung and Scanlon (2001) [4], Muhammad Khalil,Umair Khalil, Zahoor ulHaq (2019) [5], Royati Abdul Sahaa, Ahmad Fauzi Mohd Ayubb, Rohani Ahmad Tarmizic (2010) [6], who have observed the positive impact of the use of software on students' learning and understanding of mathematics.

The teacher must be an artist in his mission, using different techniques to teach and to solve mathematical problems.

\section{Solving A Geometrical Locus Problem Using GeoGebra Software}

\section{Statement of the Problem:}

consider the following problem: "In the plane, $A B C$ is a triangle of orthocenter $H$. Determine the geometrical locus $L$ of the points $H$ when $C$ moves on a fixed line $(D)$ ".

\section{ANSWER}

Three possible cases can be considered in relation to the line $(D)$ :

- $(D)$ is perpendicular to the abscissa axis at a point $I$. 
- $(D)$ is perpendicular to the ordinate axis at a point $J$.

- $(D)$ is an oblique line.

\subsection{First Case: $(D)$ is Perpendicular to the Abscissa Axis at A Point I}

- Construction Protocol:

We launch GeoGebra software and we build the following objects: point $A$, intersection of the coordinate axes, point $B$, point of the abscissa axis, a point $I$, point of the abscissa axis, the line $(D)$ which passes through the point $I$ and perpendicular to the abscissa axis, a point $C$ of the straight line $(D)$, the triangle $A B C$, the orthocenter $H$ of the triangle $A B C$. Using the command: geometrical locus, we obtain he geometrical locus $L$ of the point $H$ when the point $C$ varies on the line $(D)$ we find: $L=(D)$ Figure 2. We move the point $I$ along the $\mathrm{x}$-axis, we always find $L=(D)$.

If we move point $C$ by the mouse, point $H$ describes the line $(D)$, point $H$ is not defined when $A, B$ and $C$ are aligned.

When we move point $I$ along the abscissa axis, we always find the geometrical locus of $H$ is the line $(D)$.

- Conclusion: in this case, the geometrical locus of the points $H$ is the line $(D)$.

\subsection{Second Case: $(D)$ is Perpendicular}

to the Ordinate Axis at A Point J

- Construction Protocol:

We launch the GeoGebra software and we build the following objects: point $A$, intersection of the coordinate axes, point $B$, point of the abscissa axis, a point $J$, point of the $\mathrm{Y}$ axis, the line $(D)$ which passes through the point $J$ and perpendicular to the $\mathrm{Y}$ axis, a point $C$ of the straight line $(D)$, the triangle $A B C$

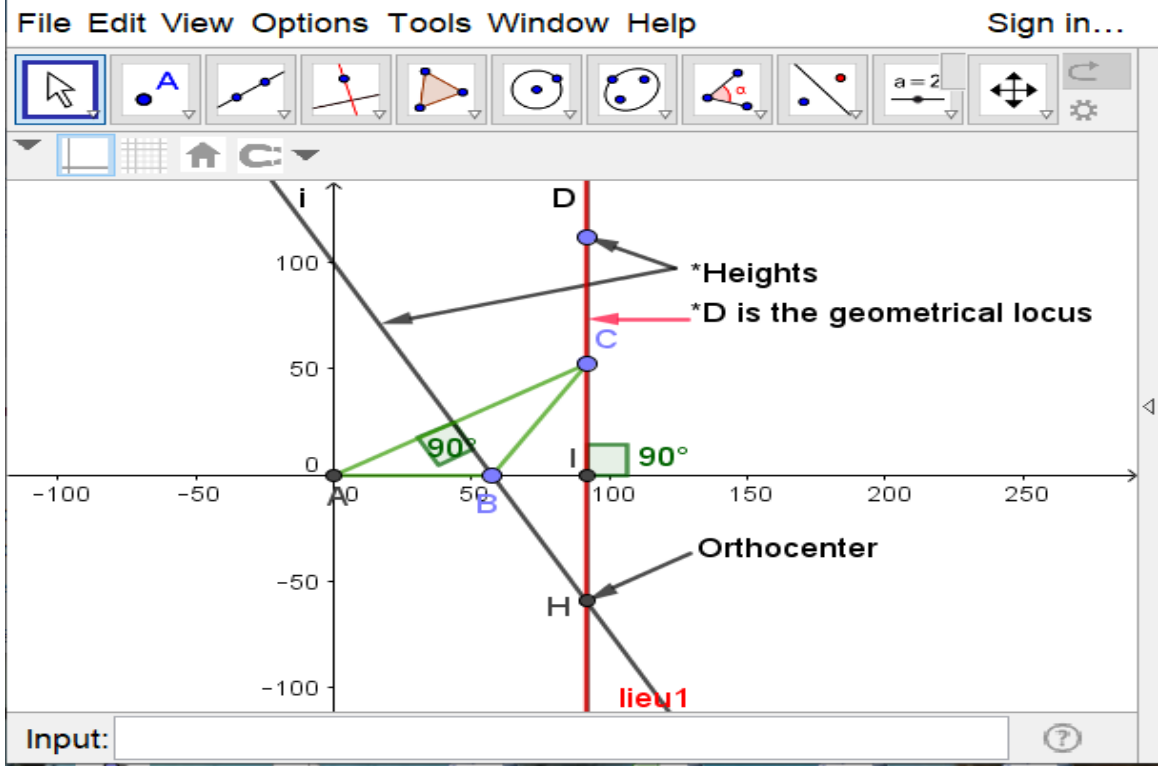

Figure 2. $(D)$ is perpendicular to the abscissa axis at a point $I$ and $L=(D)$. 
the orthocenter $H$ of the triangle $A B C$. Using the command: geometrical locus, we obtain the geometrical locus $L$ of the point $H$ when the point $C$ varies on the line $(D)$ Figure 3 or Figure 4.

We move point $J$ along the ordinate axis to see the shape of the geometrical locus relative to the position of the line $(D)$.

We deduce that:

- If the ordinate of point $J$ is positive, the geometrical locus is concave, Figure 3.

- If the ordinate of point $J$ is zero, point $H$ is not defined

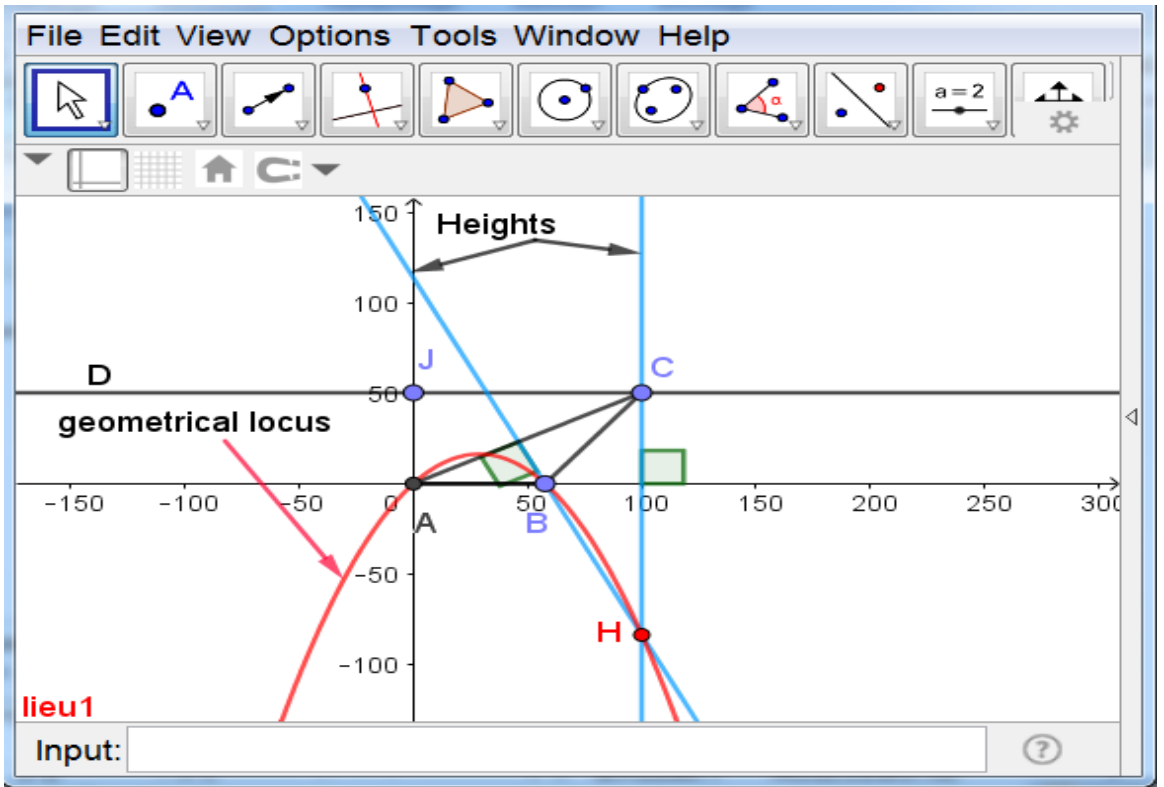

Figure 3. The ordinate of point $J$ is positive, the geometrical locus is concave.

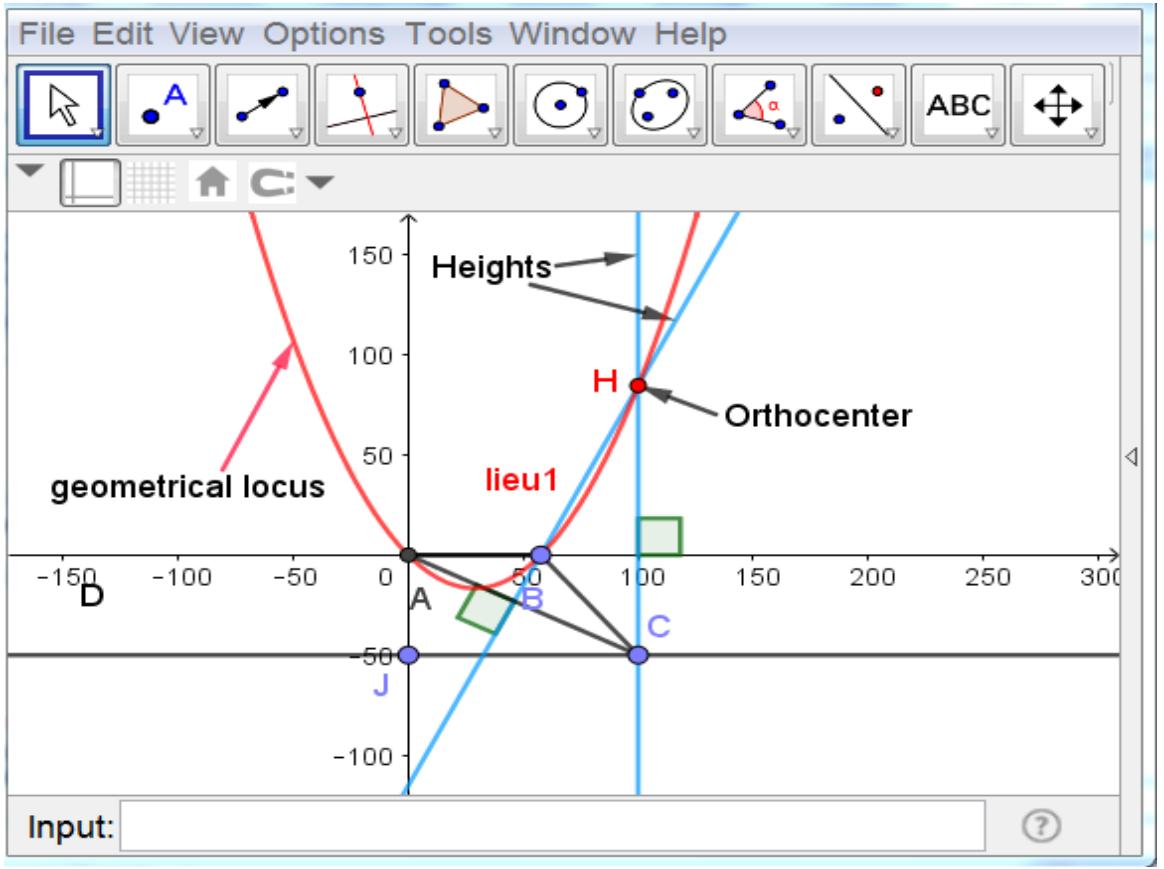

Figure 4. The ordinate of point $J$ is negative, the geometrical locus is convex. 
- If the ordinate of point $J$ is negative, the geometrical locus is convex, Figure 4. Note: We have the impression that the geometrical locus is a parabola.

\subsection{Third Case: (D) is An Oblique Line}

In this case, the line $(D)$ is oblique, it intersects the abscissa axis at point $I$, and it makes an angle $\alpha$ with the abscissa axis; $\alpha$ varies from $0^{\circ}$ to $180^{\circ}$.

- Construction Protocol

We launch the GeoGebra software and we build the following objects: point $A$, intersection of the coordinate axes, point $B$, point of the abscissa axis, a point $I$, point of the x-axis, the angle $\alpha$ by a cursor, the line $(D)$ which makes the angle $\alpha$ with the x-axis passing through the point $I$, a point $C$ of the line $(D)$, the triangle $A B C$, the orthocenter $H$ of the triangle $A B C$, the geometrical locus of point $H$ when point $C$ varies. We represent the angle $\alpha$ by a cursor, point $I$ is displaced along the abscissa axis. we have captured Figures 5-14:

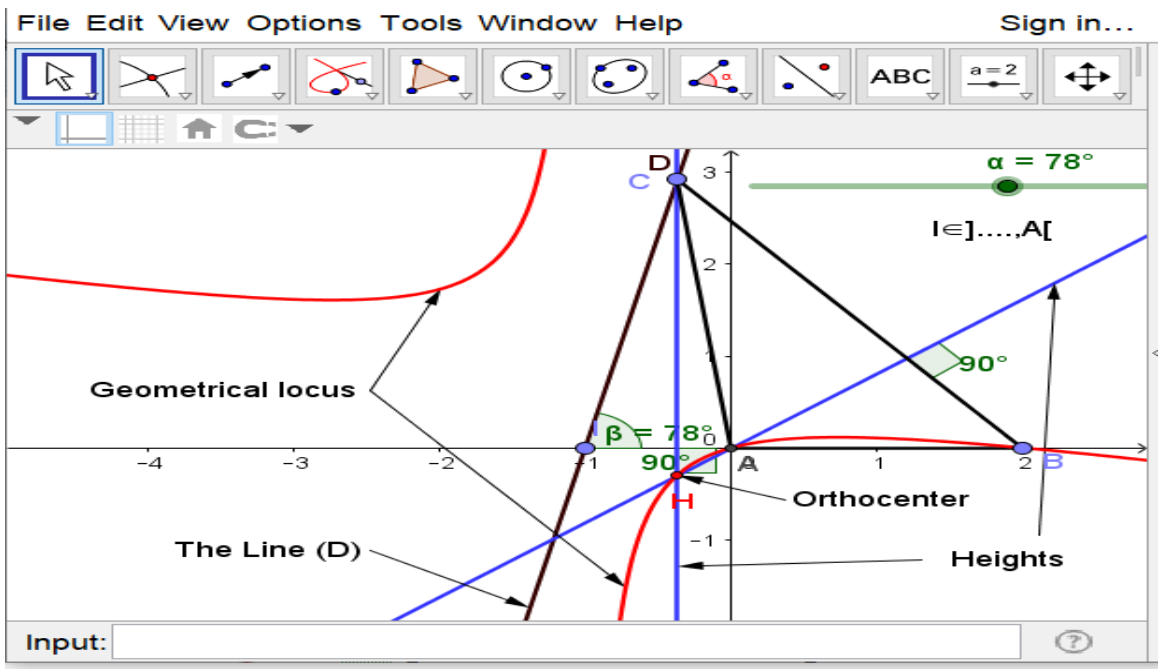

Figure 5. Point $I$ to the left of point $A$.

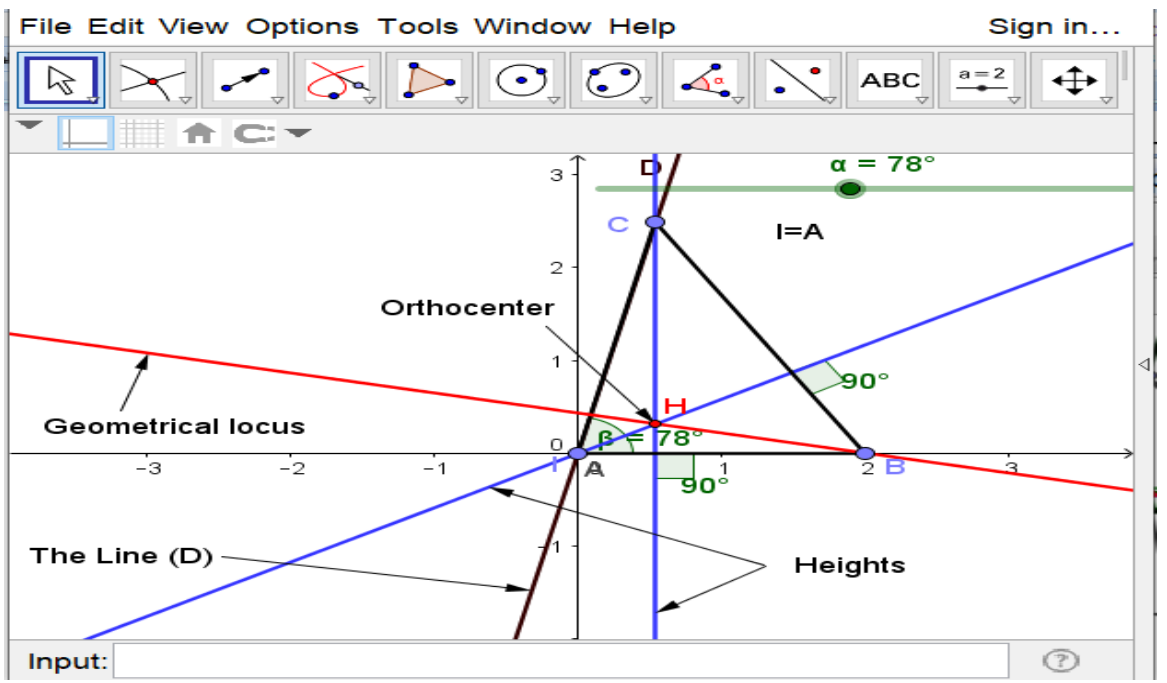

Figure 6. $I=A$. 


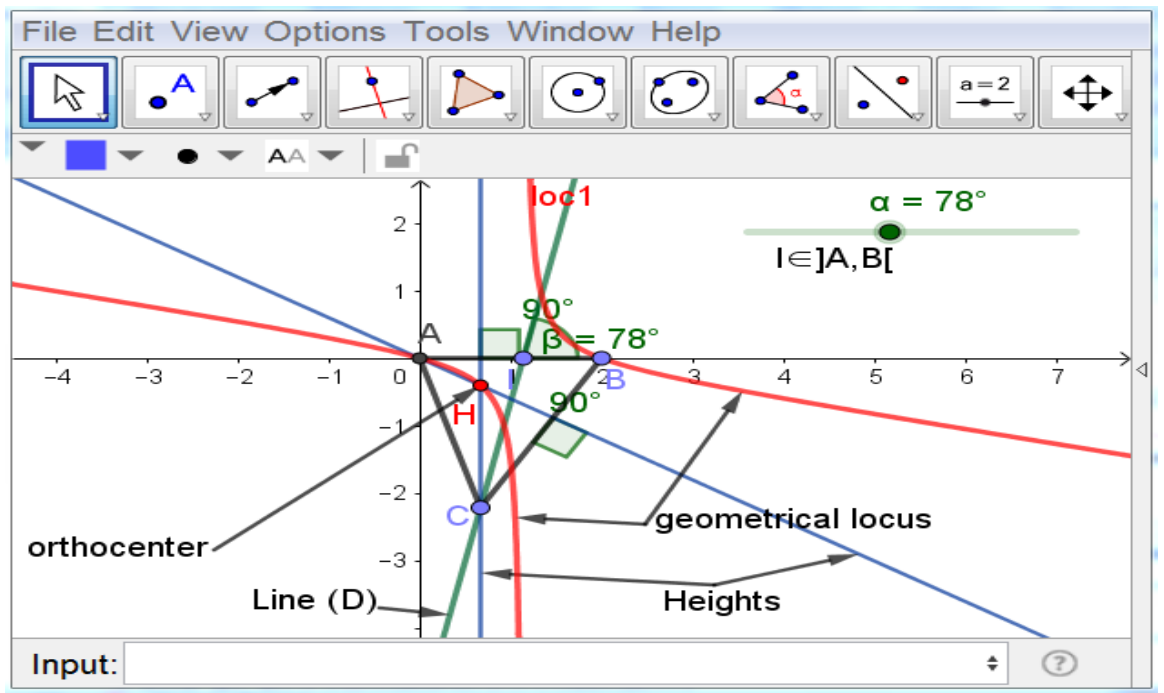

Figure 7. I belongs to $] A B[$.

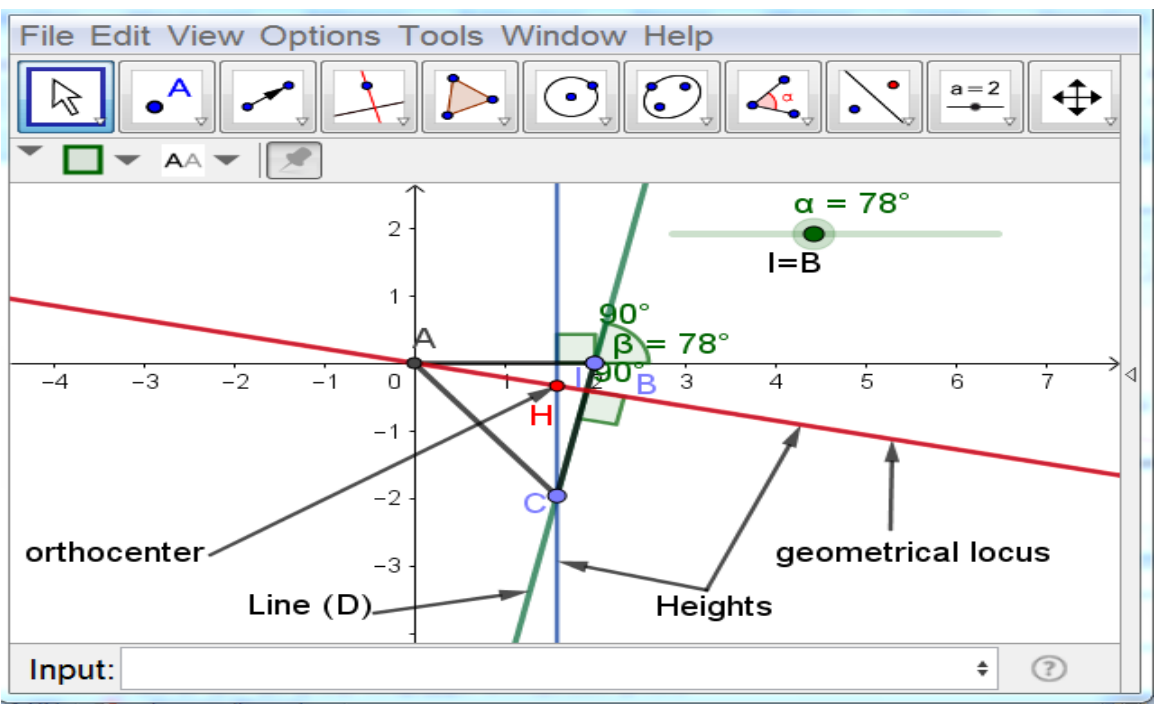

Figure 8. $I=B$.

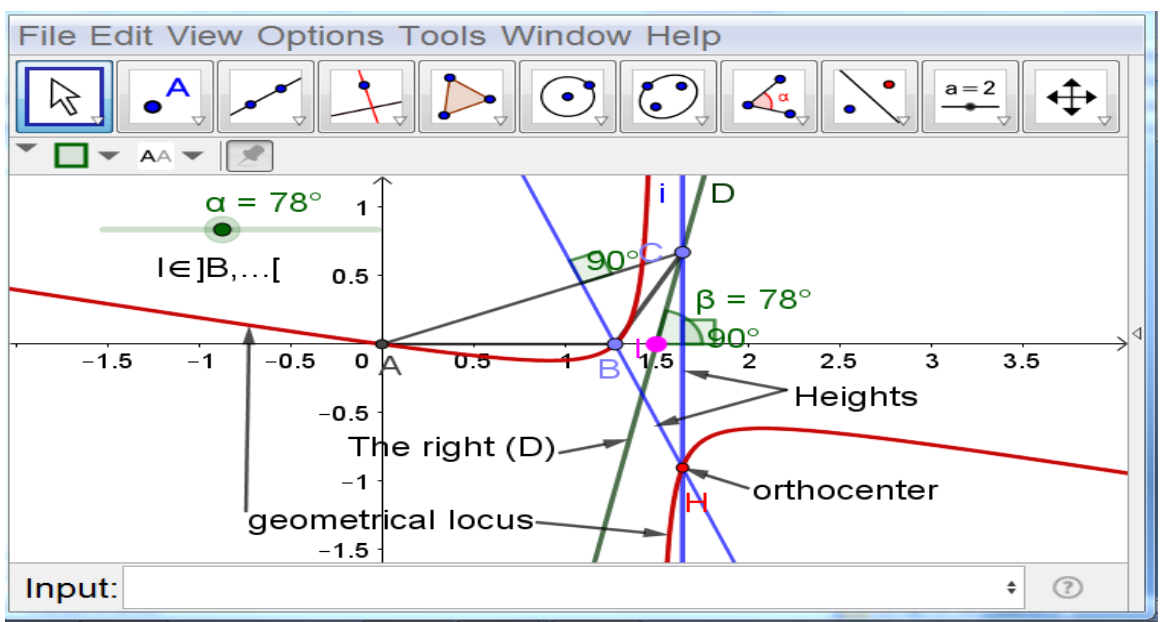

Figure 9. I to the right of $B$. 


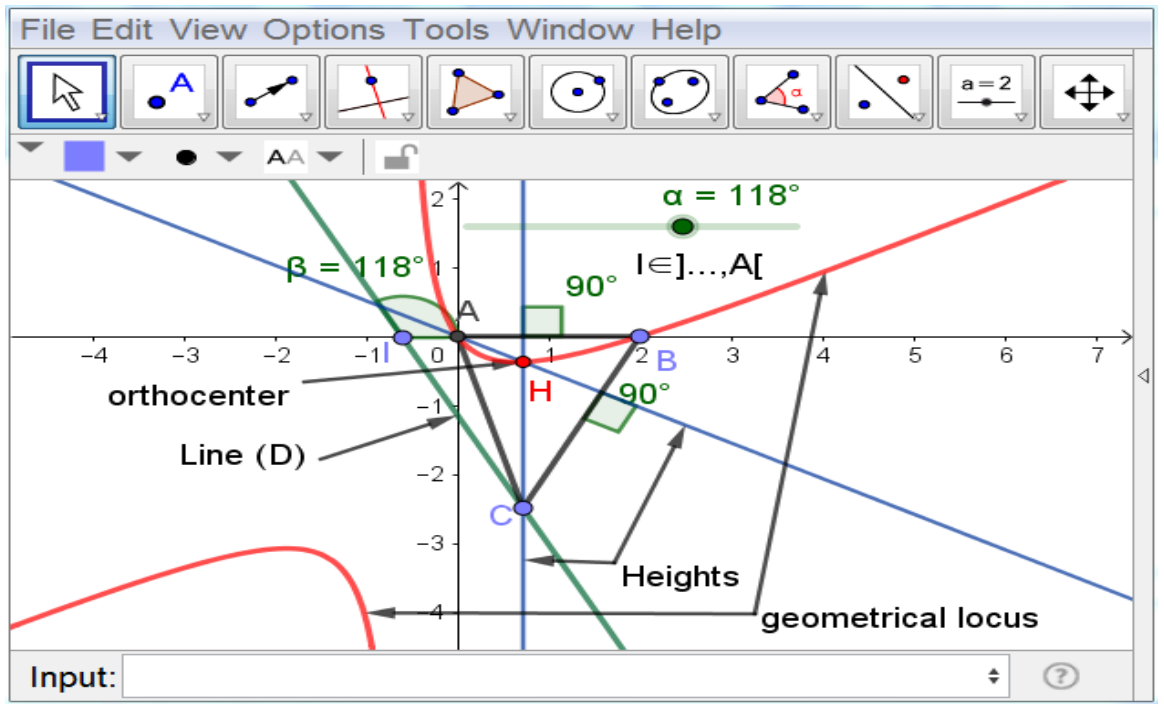

Figure 10. Point $I$ to the left of point $A$.

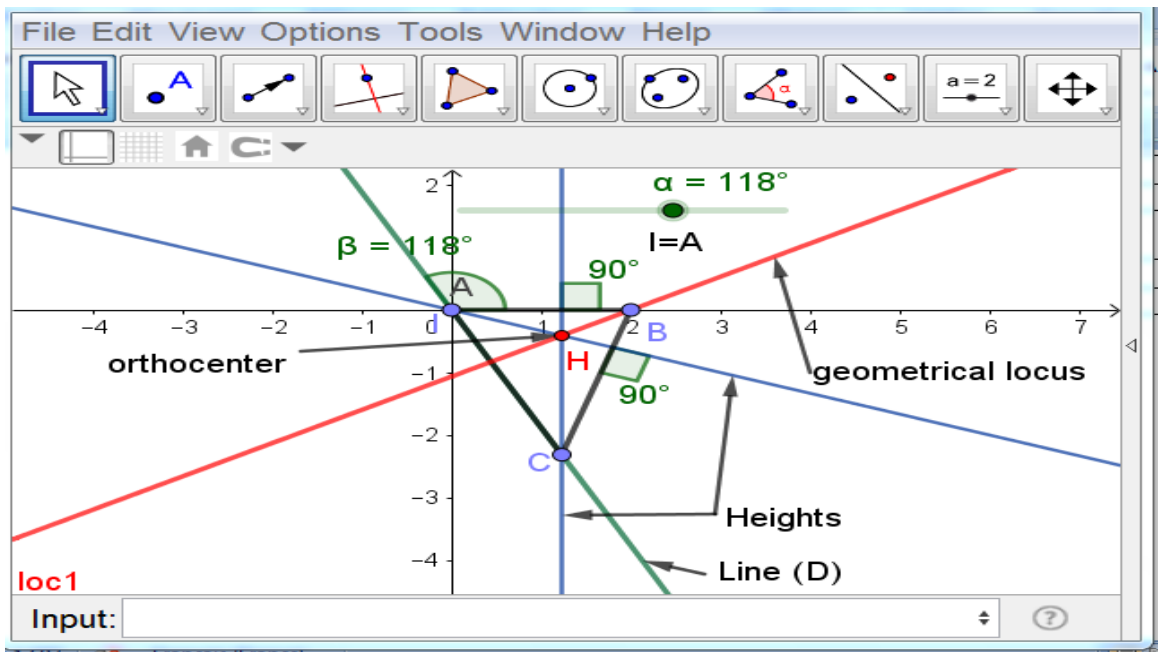

Figure 11. $I=A$.

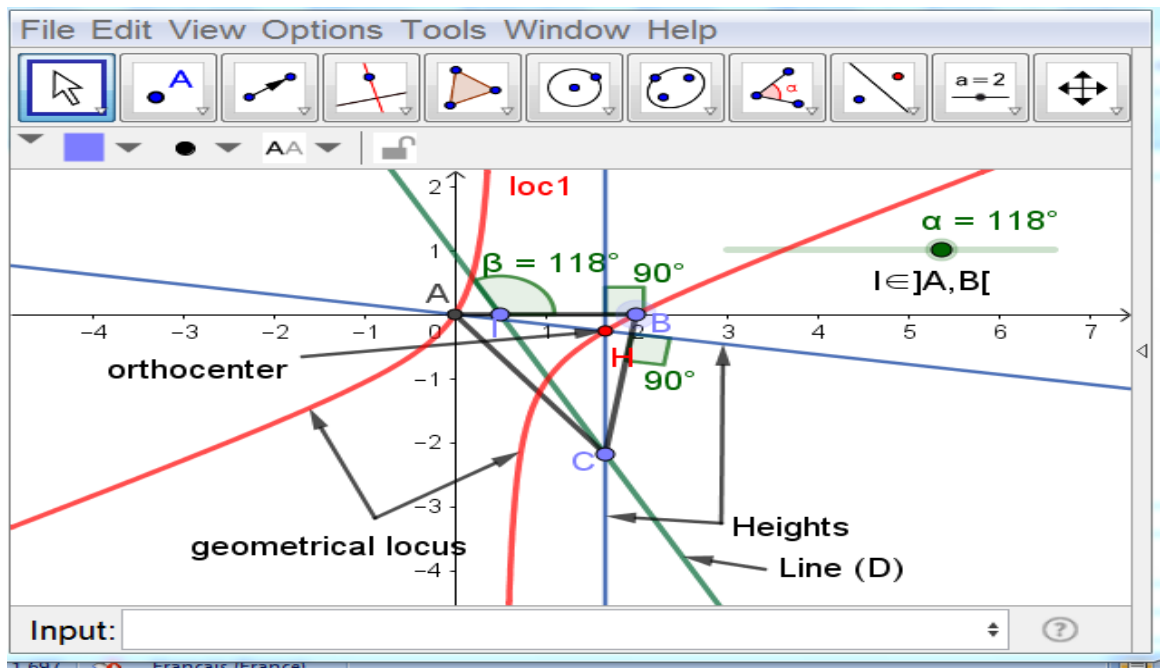

Figure 12. I belongs to $] A B[$. 


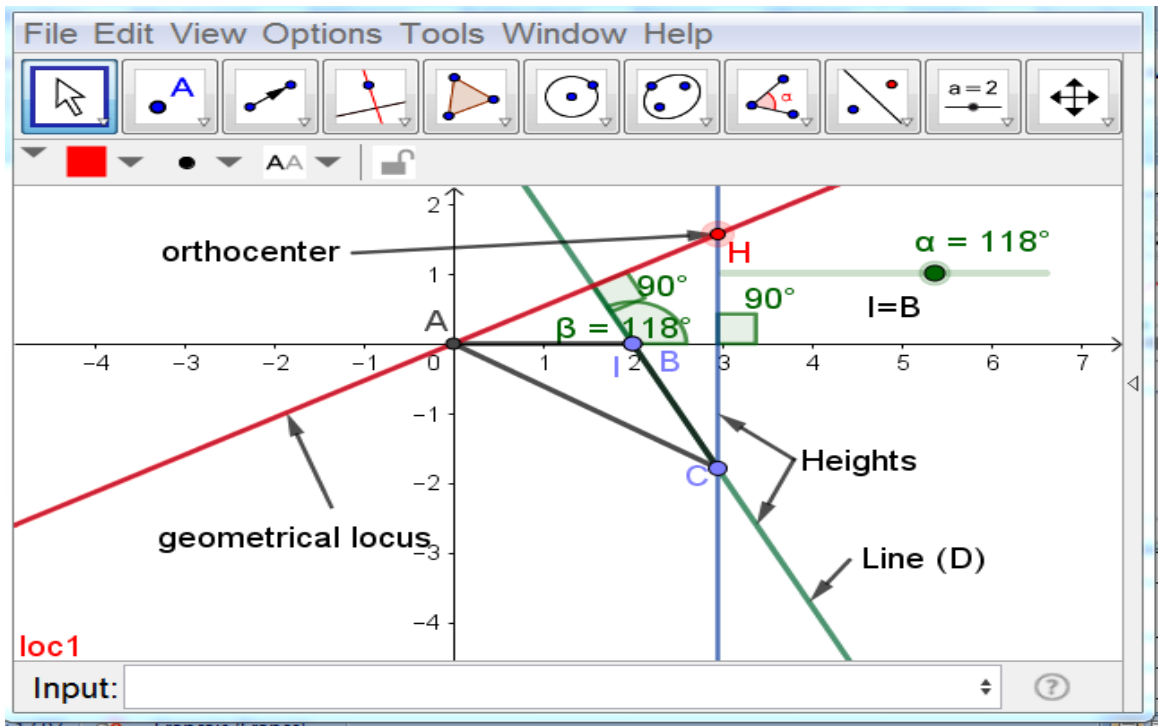

Figure 13. $I=B$.

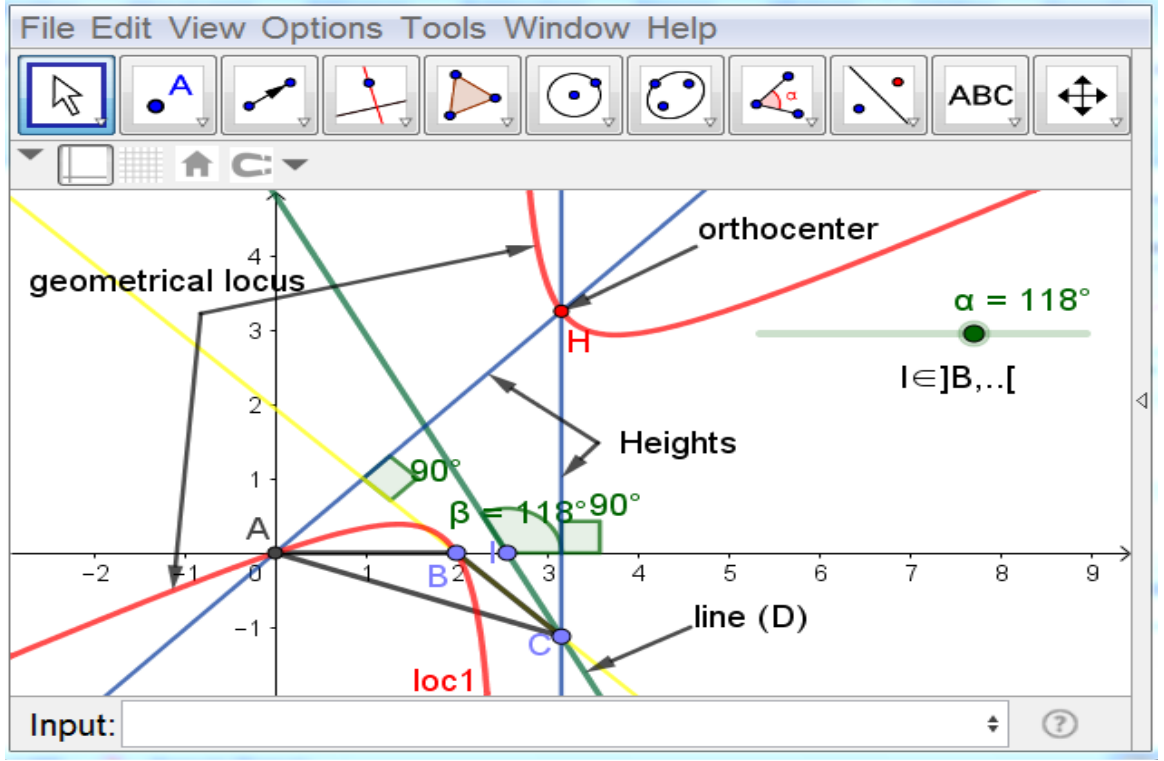

Figure 14. $I$ to the right of $B$.

"Five figures when the slope of the line is positive and Five other figures when the slope of the line is negative".

- line $(D)$ has a positive slope:

- The line $(D)$ has a negative slope.

Remarks and conclusions:

- If $I=A$, the geometrical locus is the line perpendicular to $(D)$ passing through $B$, Figure 6 and Figure 11 .

- If $I=B$, the geometrical locus is the line perpendicular to $(D)$ passing through $A$, Figure 8 and Figure 13.

- If $I \neq A$ and $I \neq B$

1) The geometric locus are graphic representations of the numerical functions, 
these functions are defined continuous and differentiable on the set of real numbers $I R$ except in a, where a is the abscissa of point $I$

2) If the slope of the line $(D)$ is positive and if I belongs to the segment $[A B]$, the geometrical locus is decreasing, Figure 7.

3 ) If the slope of the line $(D)$ is positive and if $I$ belongs to the half-line (., $A)$, or $(B, .$.$) , the geometrical locus presents a minimum then a maximum, Figure 5$ and Figure 9.

4) If the slope of the line $(D)$ is negative and if $I$ belongs to the segment $[A B]$, the geometrical locus is increasing Figure 12.

$5)$ If the slope of the line $(D)$ is negative and if $I$ belongs to the half-line $(. ., A)$, or $(B, .$.$) , the geometrical locus presents a maximum then a minimum, Figure 10$ and Figure 14.

6) All the curves pass through points $A$ and $B$, in fact: the vertical passing through point $A$ intersects the line $(D)$ at a point $C$, so $A$ is the orthocenter of the triangle $A B C$, similarly for point $B$.

\section{Analytical Geometry}

\subsection{Some Preliminary Notes}

Definition: A geometrical locus is a set of points satisfying the conditions, given by a mathematical problem.

By the introduction the Cartesian coordinate system, the points are characterized by their coordinates and the sets of points are characterized by Cartesian equations. Geometric questions are translated algebraically, which sometimes facilitates solutions.

René Descartes (1596-1650) is the first to introduce two-dimensional Cartesian landmarks [7] [8] and Lagrange (1736-1813) is the first to use the equations of lines and planes [9]. Monge (1746-1818) invented the concept of vectors [9].

We present here two methods, which are often used to determine a geometrical place.

\section{The translation method}

It consists of:

- Choose an orthonormal coordinate system, so that the data have simple equations.

- Graph the data.

- Translate data and conditions by algebraic relationships.

- We obtain a relation, or an equation verified by the coordinates of the points of the geometrical locus.

- We study the converse and we trace the place in question.

The generator method:

The generatrices are curves, which contain the point $\mathrm{P}$ whose geometrical locus is sought. The method consists of:

- Choose a reference, it is not necessarily orthonormal.

- Graph the data. 
- Specify the equations of the fixed lines and the coordinates of the fixed points.

- Identify the generators.

- Find the equations of the generators.

- Eliminate the parameter between the two generators, thus obtaining the equation of the geometrical locus.

- Analyze and build the geometric locus.

\subsection{Resolution of Problem}

In 1945 George Polya published his famous book How to Solve It; more than a million copies have been sold; this book has been translated into 17 languages. In this book, George Polya identifies four basic principles for maximum luck in solving a problem, which are [10]:

- First Principle: Understand the problem.

- Second Principle: Devise a plan.

- Third Principle: Carry out the plan.

- Fourth Principle: Look back.

But there is problem and problem, for our problem, we will follow other steps without forgetting the advice of George Polya.

Formulation of the Problem:

Problem: "In the plane, $A B C$ is a triangle, of orthocenter $H$. Determine the geometrical locus L of the points $H$ when $C$ moves on a fixed line $(D)$ ".

We consider an orthonormal coordinate system, $\left(A, \overrightarrow{A B}, \overrightarrow{A B^{\prime}}\right)$.

Let $H$ be the orthocenter of the triangle $A B C$, its coordinates are $(r, s)$.

\section{Mathematization of the Problem}

- First case: $(D)$ is perpendicular to the abscissa axis at a point $I(a, 0)$.

The line $(D)$ is a height, so $x=a$ is the abscissa of $H$, its ordinate varies from $-\infty$ to $+\infty$ when the point $C$ varies along the line $(D)$, therefore $(D)$ is the geometrical locus of $H$, Figure 2 .

- Second case: $(D)$ is perpendicular to the ordinate axis at a point $J(0, a)$.

The equation of the line $(D)$ is $y=a, C$ is a point on the line $(D)$, its coordinates are $(r, a)$ with $r$ is a real varies. Let $H$ be the orthocenter of the triangle $A B C$, its coordinates are $(r, a)$. Point $H$ checks:

$$
\overrightarrow{B H} \cdot \overrightarrow{A C}=0
$$

So:

$$
(r-1)(r-0)+(s-0)(a-0)=0
$$

So

$$
g(x)=x(1-x) / a
$$

The Equation (3) is the equation of a parable of vertex $E(1 / 2,1 / 4 a)$ and axis of symmetry $x=a$, Figure 3 and Figure 4 .

- Third case $(D)$ is an oblique line.

Of coordinate system, the line $(D)$ is oblique, so it intersects the abscissa axis at a point $I(a, 0)$ where $a$ is a real number. The equation of the line $(D)$ is 
written

$$
y=(x-a) \operatorname{tg}(\alpha) .
$$

With $\alpha$ is an element of the interval $] 0, \pi / 2[\cup] \pi / 2, \pi[$.

$C$ is a point on the line $(D)$, its coordinates are $(r,(r-a) \operatorname{tg}(\alpha))$ with $r$ is a real number.

Let $H$ be the orthocenter of the triangle $A B C$, its coordinates are $(r, s)$

\section{Mathematization of the Problem}

Point $H$ checks

$$
\overrightarrow{B H} \cdot \overrightarrow{A C}=0
$$

Using Cartesian coordinates, we find the coordinates $(r, s)$ of the orthocenter $H$ verify:

$$
r(r-1)+s(r-a) \operatorname{tg}(\alpha)=0
$$

\section{Resolution of problem:}

- First case: if $a=0$, the Equation (6) becomes

$$
r(r-1+\operatorname{stg}(\alpha))=0 \text {. }
$$

Or, $r$ varies, so

$$
s=(1-r) / \operatorname{tg}(\alpha)
$$

That we write

$$
y=(1-x) / \operatorname{tg}(\alpha)
$$

So, the geometrical locus is a line which passes through point $B$, Figure 6 and Figure 11.

- Second case: if $a=1$, the Equation (6) becomes:

$$
(r-1)(r+\operatorname{stg}(\alpha))=0 .
$$

Or, $r$ varies, so

$$
s=-r / \operatorname{tg}(\alpha) \text {. }
$$

That we write

$$
y=-x / \operatorname{tg}(\alpha)
$$

It is a line, which passes through point $A$, Figure 8 and Figure 13.

Third case: $a \neq 0$ and $a \neq 1$, The numbers $a$ and $\alpha$ are parameters. $\alpha \neq 0$ because $A B C$ is an unfastened triangle. So:

$$
s=r(1-r) /(r-a) \operatorname{tg}(\alpha) \text {. }
$$

Hence, the geometric locus of the orthocenter $H$ is the curve $C(a, \alpha)$ representing the function

$$
g_{(a, \alpha)}(x)=x(1-x) /(x-a) \operatorname{tg}(\alpha) .
$$

Note that the curves $C(a, \alpha)$ and $C(a, \pi-\alpha)$ are symmetrical with respect to the abscissa axis because $\operatorname{tg}(\alpha)=-\operatorname{tg}(\pi-\alpha)$, also note that: $M(x, y)$ 
is a point of $C(a, \pi / 4)$ equivalent to $M^{\prime}(x, y / \operatorname{tg}(\alpha))$ is a point of $C(a, \alpha)$ because $\operatorname{tg}(\pi / 4)=1$.

We are only interested in the representation of the curve $C(a, \pi / 4)$

$$
g_{(a, \pi / 4)}(x)=x(1-x) /(x-a)
$$

The function $g_{(a, \pi / 4)}$ is a defined, continuous and differentiable on ]$-\infty, a[\cup] a,+\infty[$ and its derivative is:

$$
g_{(a, \pi / 4)}^{\prime}(x)=\left(-x^{2}+2 a x-a\right) /(x-a)^{2}
$$

$g_{(a, \pi / 4)}^{\prime}(x)=0$ if and only if the discriminant $a^{2}-a$ is positive, its roots are $x_{2}=-a-\sqrt{a^{2}-a}$ and $x_{2}=-a+\sqrt{a^{2}-a} .\left(a^{2}-a<0\right.$ if $0<a<1$ Otherwise $\left.a^{2}-a>0\right)$.

If $0<a<1, g_{(a, \pi / 4)}^{\prime}(x)<0$, then the function $g_{(a, \pi / 4)}$ is strictly decreasing.

The table of variation (Table 1 and Table 2):

The line $x=a$ is an asymptote; to find the oblique asymptotes, we change the variable: $x=X+a$ we find: $x(1-x) /(x-a)=-x-a+1+\left(a-a^{2}\right) /(x-a)$, We deduce that the line $y=-x-a+1$ is an asymptote which is below the curve $C(a, \pi / 4)$ on $]-\infty, a[$ and above the curve $C(a, \pi / 4)$ on $] a,+\infty[$.

\section{Graphical representation using GeoGebra software:}

We launch the GeoGebra software. The results were shown as Figures 15-17.

We define the cursor, we enter the function $g_{(a, \pi / 4)}(x)$ and the equations of the asymptotes the line $y=-x-a+1$ and the line $x=a$.

IF $a<0$ or $a>1, g_{(a, \pi / 4)}^{\prime}$ vanishes at $x_{1}$ and $x_{2}$ moreover it is positive on $] x_{1}, x_{2}\left[\right.$ because $x_{1}<a<x_{2}$ and $\left(-x^{2}+2 a x-a\right)$ is positive for $x=a$.

\section{Conclusions}

To conclude, the adoption of dynamic geometry via GeoGebra in the resolution of a problem of the geometrical locus allowed to emit and formulate a conjecture which was used as a basis for a resolution of this problem by the analytical geometrics.

Table 1. The table of variation of $g_{(a, \pi / 4)}$ for $0<a<1$.

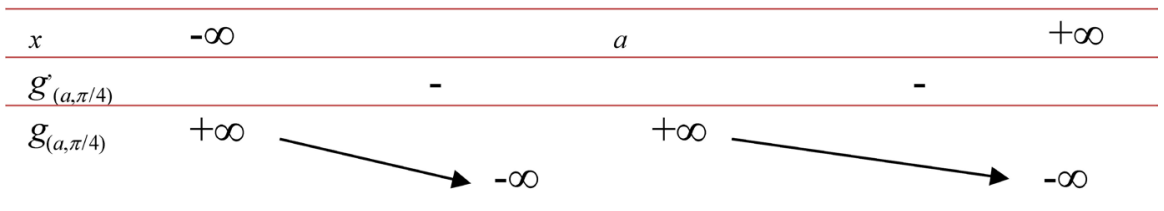

Table 2. The table of variation of $g_{(a, \pi / 4)}$ for $a<0$ or $a>1$.

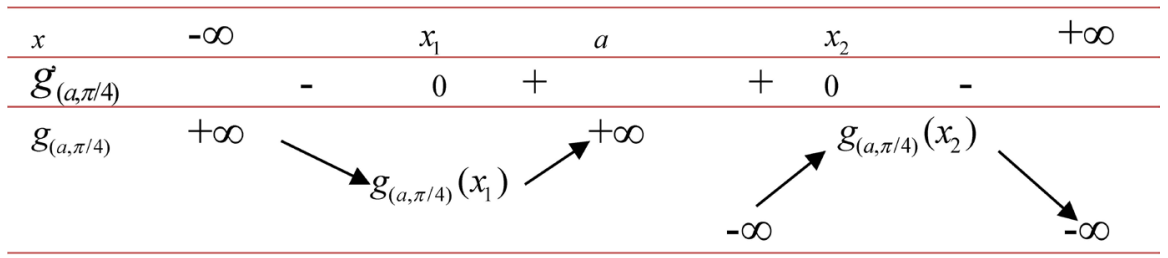




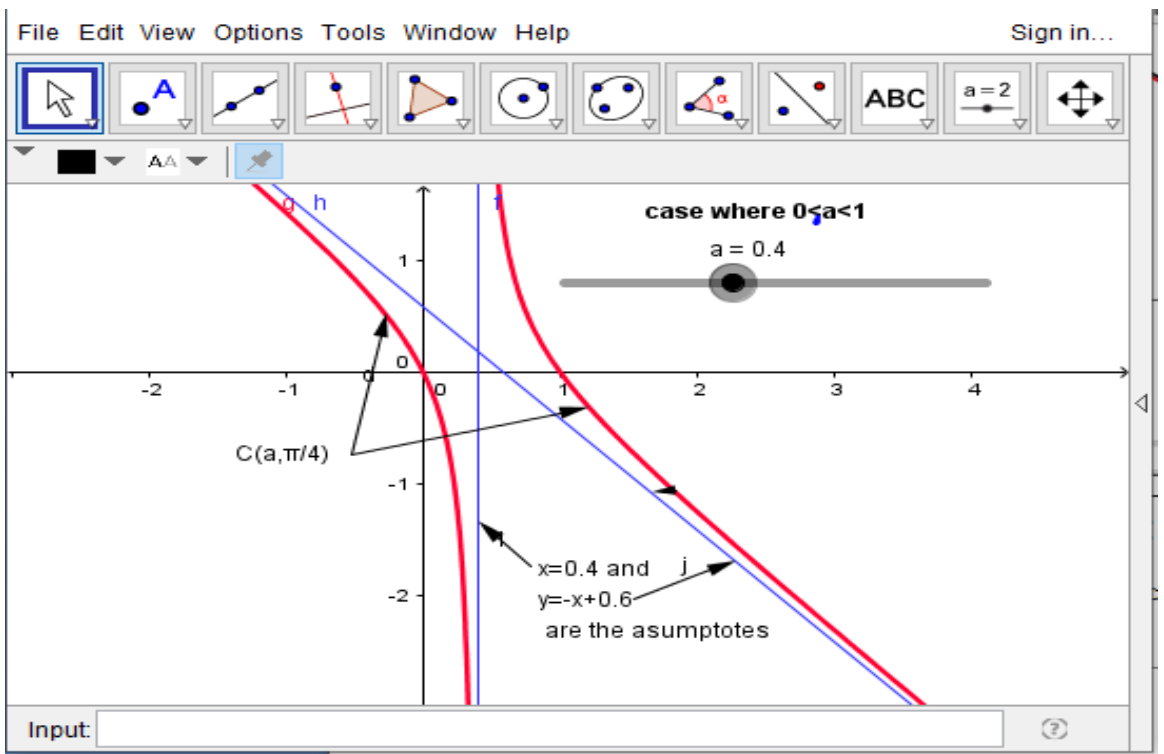

Figure 15. Graphical representation of $g_{(a, \pi / 4)}$ for $0<a<1$.

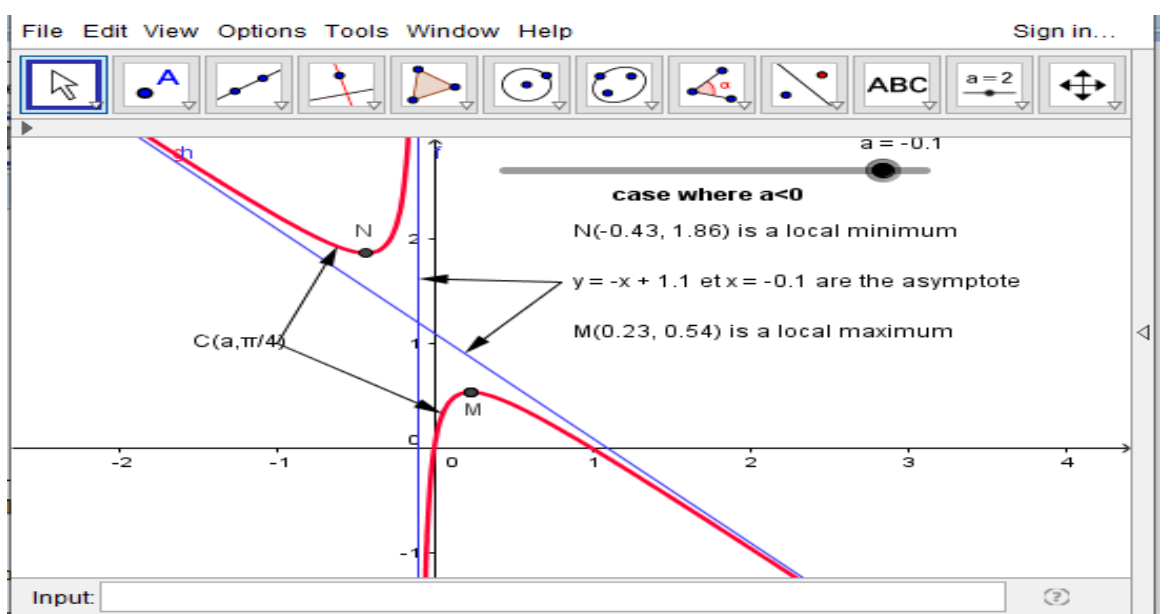

Figure 16. Graphical representation of $g_{(a, \pi / 4)}$ for $a<0$.

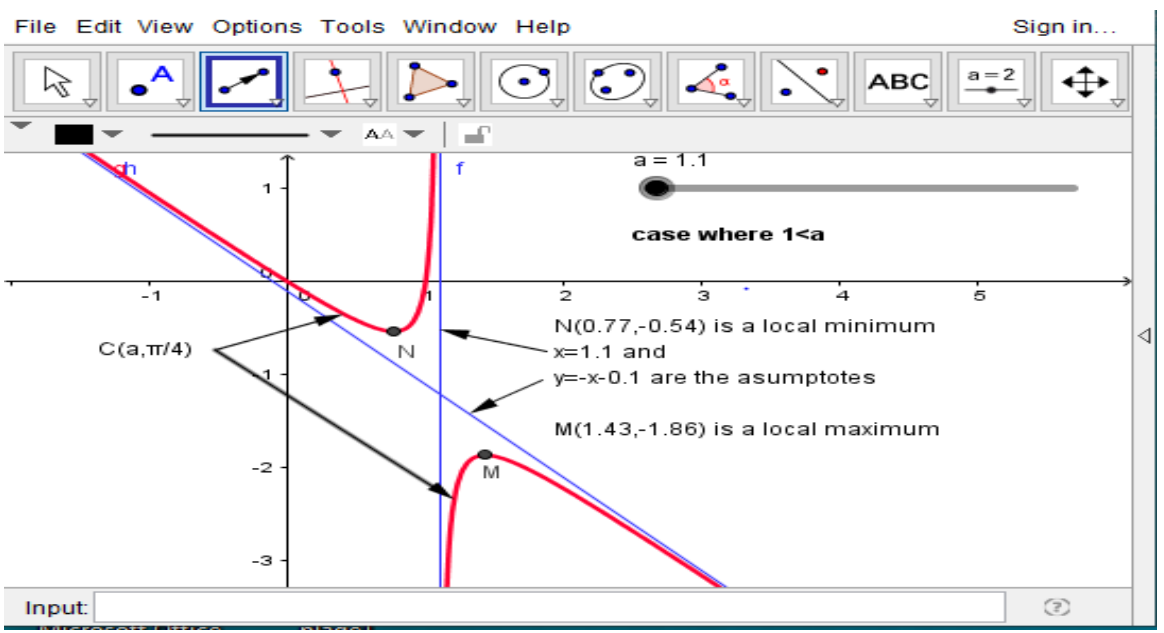

Figure 17. Graphical representation of $g_{(a, \pi / 4)}$ for $a>1$. 
Textbook authors are expected to take advantage of this software to make students more attractive and motivated to examine and study geometric locus problems.

Software that will appeal to teachers and students of mathematics, it should add a playful side to learning geometry and algebra.

The participation of future teachers clearly demonstrates the pedagogical effectiveness of GeoGebra compared to traditional construction tools.

The efficient use of the computer is appreciated, as it promotes mathematics for all. This is why teachers trained in mathematics should develop the skills and abilities necessary to take advantage of available technological resources [11].

The new method of teaching and learning in mathematics, using GeoGebra software in the teaching and learning process, results in a much greater increase in the level of knowledge and skills in mathematics than the traditional method used in this process [12].

\section{Now Let's Solve This Problem}

Let ( $d$ ) be a fixed line of the plane and a point $A$ not belonging to $(d)$.

For each point $M$ of the line (d) we consider the circles (c) with center $M$ passing through $A$ and (c) with center $A$ passing through $M$.

What are the geometric locus $\left(L_{1}\right)$ and $\left(L_{2}\right)$ of the points $M_{1}$ and $M_{2}$, intersection of the two circles?

\section{Conflicts of Interest}

The authors declare no conflicts of interest regarding the publication of this paper.

\section{References}

[1] Alias, M. (2000) Spatial Visualisation Ability and Problem Solving in Civil Engineering. Ph.D. Thesis, School of Educational Studies, University of Surrey, Guildford.

[2] Sahaa, R.A., Ayubb, A.F.M. and Tarmizi, R.A. (2010) The Effects of GeoGebra on Mathematics Achievement: Enlightening Coordinate Geometry Learning. Procedia Social and Behavioral Sciences, 8, 686-693. https://core.ac.uk/download/pdf/82664417.pdf

[3] Freiman, V., Martinovic, D. and Karadag, Z. (2009) Decouvrir le potentiel educatif du logiciel dynamique GeoGebra. Bulletin $A M Q$, XLIX. https://www.amq.math.ca/ancien/bulletins/dec09/Article-GeoGebra.pdf

[4] Hennessy, S., Fung, P. and Scanlon, E. (2001) The Role of the Graphic Calculator in Mediating Graphing Activity. International Journal of Mathematics Education for Science and Technology, 32, 267-290. https://doi.org/10.1080/00207390010022176

[5] Khalil, M., Khalil, U. and ulHaq, Z. (2019) GeoGebra as a Scaffolding Tool for Exploring Analytic Geometry Structure and Developing Mathematical Thinking of Diverse Achievers. International Electronic Journal of Mathematics Education, 14, 427-434. https://doi.org/10.29333/iejme/5746

[6] Tarmizi, R.A. (2010) The Effects of GeoGbra on Mathematics Achievement Enligh- 
tening Coordinate Geometry Learning.pdf.

[7] https://debart.pagesperso-orange.fr/geometrie/

[8] https://www.storyofmathematics.com/17th descartes.html

[9] https://en.wikipedia.org/wiki/Joseph-Louis Lagrange\#Other mathematical work

[10] https://math.berkeley.edu/ gmelvin/polya.pdf

[11] Lupu, C. (2015) The Role of Technology in the Process of Learning Mathematics. American Journal of Operations Research, 5, 89-94.

https://doi.org/10.4236/ajor.2015.52008

[12] Kllogjeri, A. and Kllogjeri, P. (2015) Statistical Inferences Supporting the Hypothesis of Teaching with GeoGebra. Open Access Library Journal, 2, e1255.

https://doi.org/10.4236/oalib.1101255 